PROCEEDINGS OF THE

AMERICAN MATHEMATICAL SOCIETY

Volume 140, Number 3, March 2012, Pages 1023-1031

S 0002-9939(2011)11343-1

Article electronically published on October 4, 2011

\title{
RANDOM GROUPS HAVE FIXED POINTS ON CAT(0) CUBE COMPLEXES
}

\author{
KOJI FUJIWARA AND TETSU TOYODA
}

(Communicated by Ken Ono)

\begin{abstract}
We prove that a random group has fixed points when it isometrically acts on a $\operatorname{CAT}(0)$ cube complex. We do not assume that the action is simplicial.
\end{abstract}

\section{INTRODUCTION}

In 4, Gromov introduced random groups of the graph model to show the existence of groups which cannot embed uniformly into Hilbert spaces (see Theorem 1.8). He also showed that when a random group of the graph model isometrically acts on finite or infinite dimensional Hadamard manifolds, there exist common fixed points. Silberman [12] provided details of the argument for the case of Hilbert spaces (see Theorem 1.3 for the precise statement).

Definition 1.1 (random group of Gromov's graph model). Let $G=(V, E)$ be a finite graph such that $V$ is the set of vertices and $E$ is the set of edges. Orient the edges $E$ arbitrarily. Fix $k$ alphabets $s_{1}, \ldots, s_{k}$. For each $e \in E$, choose an element $a(e)$ independently, uniformly at random from $\left\{s_{1}, \ldots, s_{k}, s_{1}^{-1}, \ldots, s_{k}^{-1}\right\}$. Let $c=e_{1}^{\epsilon_{1}} \cdots e_{n}^{\epsilon_{n}}, e_{i} \in E$ be a cycle in $G$, where $\epsilon_{i}=1$ or -1 , and $e^{-1}$ means the edge $e$ with the orientation reversed. The cycle $c$ defines a random word $a(c)=$ $a\left(e_{1}\right)^{\epsilon_{1}} \cdots a\left(e_{n}\right)^{\epsilon_{n}}$ on $\left\{s_{1}, \ldots, s_{k}, s_{1}^{-1}, \ldots, s_{k}^{-1}\right\}$. Let $R_{G}$ be the set of the random words $a(c)$ for all cycles in $G$. In this way, we obtain a probability distribution over groups $\Gamma(G)=\left\langle s_{1}, \ldots, s_{k} \mid R_{G}\right\rangle$. We say that $\Gamma(G)$ is the random group associated to $G$ with $k$ generators.

Properties of the random group $\Gamma(G)$, for example, infinite, word-hyperbolic, or property $(\mathrm{T})$, etc., become random variables.

Definition 1.2 (sequence of expanders). A sequence of expanders is a sequence $\left\{G_{n}=\left(V_{n}, E_{n}\right)\right\}$ of finite graphs which satisfies the following properties:

(1) The number of vertices of $G_{n}$ goes to infinity as $n$ goes to infinity.

(2) There exists $d$ such that $\operatorname{deg}(v) \leq d$ for all $v \in V_{n}$ and all $n$, where $\operatorname{deg}(v)$ is the number of edges at vertex $v$.

(3) There exists $\lambda>0$ such that $\lambda_{1}\left(G_{n}\right) \geq \lambda$ for all $n$.

Received by the editors December 17, 2010 and, in revised form, December 19, 2010.

2010 Mathematics Subject Classification. Primary 53C23; Secondary 20F65, 20P05, 51F99.

The first author is supported by Grant-in-Aid for Scientific Research (No. 19340013).

(C)2011 American Mathematical Society 
In the above definition, $\lambda_{1}(G)$ is the first positive eigenvalue of the combinatorial Laplacian of $G$. For a finite graph $G=(V, E)$, the combinatorial Laplacian $\Delta_{G}$ acts on a real-valued function $f$ on $V$ as

$$
\Delta_{G} f(v)=f(v)-\sum_{u \in V \text { such that }\{v, u\} \in E} \frac{1}{\operatorname{deg}(u)} f(u), \quad v \in V .
$$

Its first positive eigenvalue $\lambda_{1}(G)$ can be computed variationally as

$$
\lambda_{1}(G)=\inf _{\phi} \frac{\sum_{\{u, v\} \in E}\|\phi(u)-\phi(v)\|^{2}}{\sum_{v \in V} \operatorname{deg}(v)\|\phi(v)-\bar{\phi}\|^{2}}
$$

where the infimum is taken over all nonconstant maps $\phi: V \rightarrow \mathbb{R}$, and $\bar{\phi}=$ $\sum_{v \in V} \frac{\operatorname{deg}(v)}{2|E|} \phi(v)$.

The girth of a graph is the minimal length of a cycle in the graph. Recall that a group $\Gamma$ has property $(\mathrm{T})$ if and only if every affine isometric action of $\Gamma$ on a Hilbert space has a common fixed point (see [3, Ch.4]).

Theorem 1.3 (Silberman [12]). If $\left\{G_{n}=\left(V_{n}, E_{n}\right)\right\}$ is a sequence of expanders, $3 \leq \operatorname{deg}(u) \leq d$ for all $u \in V_{n}$ and all $n$, and the girth of $G_{n}$ is large enough, then the group $\Gamma\left(G_{n}\right)$ has property $(T)$ with high probability.

Formally he showed that given $k \in \mathbb{N}, d \in \mathbb{N}, \lambda>0$, there exists an explicit constant $g=g(k, \lambda)$ such that if $\left\{G_{n}=\left(V_{n}, E_{n}\right)\right\}$ is a sequence of expanders such that for all $n, \lambda \leq \lambda_{1}\left(G_{n}\right)$, the girth of $G_{n}$ is at least $g$, and $3 \leq \operatorname{deg}(u) \leq d$ for all $u \in V_{n}$, then the probability for the random group $\Gamma\left(G_{n}\right)$ generated by $k$ elements to have property $(\mathrm{T})$ is at least $1-a e^{-b\left|V_{n}\right|}$, where $a, b$ are explicit and only depend on the parameters $k, d$ and $\lambda$. The statement in Theorem 1.5 and Theorem 2.2 should be understood similarly.

Izeki and Nayatani [7] introduced an invariant $0 \leq \delta \leq 1$ for a complete CAT(0) space. See Definition 2.1 for the definition of $\delta$. A recent theorem by Izeki, Kondo and Nayatani generalizes a Hilbert space (in the definition of property $(\mathrm{T})$ ) in Theorem 1.3 to a complete CAT(0) space $Y$ with $\delta(Y)<1$ (Theorem 2.2).

A cube complex is a metric polyhedral complex in which each cell is isometric to the Euclidean cube $[0,1]^{n}$, and the gluing maps are isometries. In this paper, we study the geometry of $\mathrm{CAT}(0)$ cube complexes in comparison with Euclidean and more generally Hilbert spaces, using the invariant $\delta$. The following is our main technical result.

Theorem 1.4. Let $X$ be a complete $\mathrm{CAT}(0)$ cube complex. Then we have

$$
\delta(X) \leq \frac{1}{2}
$$

Therefore Theorem 2.2 applies to complete CAT(0) cube complexes and we obtain the following.

Theorem 1.5. If $\left\{G_{n}=\left(V_{n}, E_{n}\right)\right\}$ is a sequence of expanders, $2 \leq \operatorname{deg}(u) \leq d$ for all $u \in V_{n}$ and all $n$, and the girth of $G_{n}$ is large enough, then any isometric action of $\Gamma\left(G_{n}\right)$ on a complete $\mathrm{CAT}(0)$ cube complex has a common fixed point, with high probability.

We want to emphasize that we do not assume that the actions are simplicial, that the cube complexes are locally compact, or even that they are finite dimensional. 
For simplicial actions, the conclusion is not new since it follows from Theorem 1.4 and the following theorem.

Theorem 1.6 (Niblo-Reeves, [10]). If a group with property (T) acts on a complete $\mathrm{CAT}(0)$ cube complex by simplicial isometries, then it has a common fixed point.

We note that although they assume that $X$ is finite dimensional in the paper, they are only using that $X$ is complete in the proof. But the assumption that the isometries are simplicial is essential in their argument.

We give an example of a nonsimplicial isometry without a common fixed point on a CAT (0) cube complex, which is not Euclidean. Let $P_{n}, n \in \mathbb{Z}$ be Euclidean planes with tessellations by unit squares. For each $n$, choose a base vertex $v_{n} \in P_{n}$ and join $v_{n}$ and $v_{n+1}$ by a unit edge. This is our cubical CAT(0) complex $Y$. We define a simplicial isometry on $Y, s$, as a shift by 1 , sending $\left(P_{n}, v_{n}\right)$ to $\left(P_{n+1}, v_{n+1}\right)$ and preserving the square structure. Let $r$ be an isometry of $Y$ rotating each $P_{n}$ around $v_{n}$. Generally $r$ is not simplicial. Now the composition $s r$ is a desired isometry.

We record another consequence of Theorem 1.4.

Definition 1.7. Let $\left(X, d_{X}\right)$ and $\left(Y, d_{Y}\right)$ be metric spaces. A map $f: X \rightarrow Y$ is called a uniform embedding if there exist unbounded nondecreasing functions $\rho_{1}, \rho_{2}:[0, \infty) \rightarrow[0, \infty)$ such that

$$
\rho_{1}\left(d_{X}\left(x, x^{\prime}\right)\right) \leq d_{Y}\left(f(x), f\left(x^{\prime}\right)\right) \leq \rho_{2}\left(d_{X}\left(x, x^{\prime}\right)\right),
$$

for all $x, x^{\prime} \in X$. For a sequence $\left\{\left(X_{n}, d_{X_{n}}\right)\right\}$ of metric spaces, we call a sequence of maps $f_{n}: X_{n} \rightarrow Y$ a uniform embedding if there exist unbounded nondecreasing functions $\rho_{1}, \rho_{2}:[0, \infty) \rightarrow[0, \infty)$ such that

$$
\rho_{1}\left(d_{X_{n}}\left(x, x^{\prime}\right)\right) \leq d_{Y}\left(f_{n}(x), f_{n}\left(x^{\prime}\right)\right) \leq \rho_{2}\left(d_{X_{n}}\left(x, x^{\prime}\right)\right),
$$

for all $n$ and all $x, x^{\prime} \in X_{n}$.

In 4, Gromov showed the following. This is a key step to produce a finitely generated group which does not uniformly embed in a Hilbert space.

Theorem 1.8 (Gromov, see [11). Let $\left\{G_{n}\right\}$ be a sequence of expanders. Then there is no uniform embedding of $\left\{G_{n}\right\}$ into a Hilbert space.

Using Theorem 1.1, we can adapt the proof of Theorem 1.8 to a complete CAT(0) cube complex as follows. Similar variations have been well known to experts; for example, see Kondo [8] in the case of a complete $\mathrm{CAT}(0)$ space with $\delta<1$, and also Mendel-Naor 9 .

Corollary 1.9. Let $Y$ be a complete CAT(0) cube complex. Let $\left\{G_{n}\right\}$ be a sequence of expanders. Then there is no uniform embedding of $\left\{G_{n}\right\}$ into $Y$.

As we will see in the proof of Theorem 1.4, we have a uniform control of the tangent cone of a cube complex in comparison to a Hilbert space. We ask the following question.

Question 1.10. Does a complete CAT $(0)$ cube complex always uniformly embed in a Hilbert space?

It is known that if the complex is finite dimensional, then such an embedding exists ([2]). In their argument the assumption of finite dimension is essential. Since a sequence of expanders does not uniformly embed in a CAT(0) cube complex, it seems there is no easy argument to answer this question in the negative. 
The paper is organized as follows. In Section 2, we recall the definition and basic facts about the invariant $\delta$ and quote the recent theorem by Izeki-KondoNayatani which generalizes Theorem 1.3. In Section 3, we prove Theorem 1.4 and Theorem 1.5. In Section 4, we recall the proof of Theorem 1.8 and explain how to modify it for Corollary 1.9 using Theorem 1.4.

We would like to thank G. Yu for explaining the proof of Theorem 1.8

\section{RELATION BETWEen Distortion AND $\delta$}

We assume that readers are familiar with the definition and elementary facts on CAT(0) spaces. A standard reference is the book [1]. We recall the definition of the invariant introduced by Izeki-Nayatani in [7].

Definition 2.1 ([7]). Let $Y$ be a complete CAT $(0)$ space and $\mathcal{P}(Y)$ be the space of all finitely supported probability measures $\mu$ on $Y$ each of whose $\operatorname{supports} \operatorname{supp}(\mu)$ contains at least two points. For $\mu \in \mathcal{P}(Y)$, we define

$$
0 \leq \delta(\mu)=\inf _{\phi: \operatorname{supp}(\mu) \rightarrow \mathcal{H}} \frac{\left\|\int_{Y} \phi(p) \mu(d p)\right\|^{2}}{\int_{Y}\|\phi(p)\|^{2} \mu(d p)} \leq 1,
$$

where the infimum is taken over all maps $\phi: \operatorname{supp}(\mu) \rightarrow \mathcal{H}$ with $\mathcal{H}$ a Hilbert space such that

$$
\begin{aligned}
\|\phi(p)\| & =d(p, \operatorname{bar}(\mu)), \\
\|\phi(p)-\phi(q)\| & \leq d(p, q)
\end{aligned}
$$

for all $p, q \in \operatorname{supp}(\mu)$, where $\operatorname{bar}(\mu) \in Y$ is the barycenter of $\mu$. Notice that such a map $\phi$ exists. To see that, fix a unit vector $e \in \mathcal{H}$. Define $\phi(p)=d(p, \operatorname{bar}(\mu)) e$. Then, by the triangle inequality, (2.2) is satisfied. We define the Izeki-Nayatani invariant $\delta(Y)$ of $Y$ as

$$
0 \leq \delta(Y)=\sup _{\mu \in \mathcal{P}(Y)} \delta(\mu) \leq 1 .
$$

In [7, [5, 6] it is proved by Izeki, Kondo and Nayatani that certain classes of groups must have fixed points when they isometrically act on complete CAT(0) spaces $Y$ if $\delta(Y)$ is small enough. Among these, they proved that a random group of the graph model has the fixed point property for the complete CAT $(0)$ spaces whose $\delta$ are at most some constant less than 1 .

Theorem 2.2 (Izeki-Kondo-Nayatani [6]). Let $0 \leq C<1$. If $\left\{G_{n}=\left(V_{n}, E_{n}\right)\right\}$ is a sequence of expanders, $2 \leq \operatorname{deg}(u) \leq d$ for all $u \in V_{n}$ and all $n$, and the girth of $G_{n}$ is large enough, then with high probability, any isometric action of the random group $\Gamma\left(G_{n}\right)$ on a complete $\mathrm{CAT}(0)$ space $Y$ with $\delta(Y) \leq C$ has a common fixed point.

Generally, computation of the Izeki-Nayatani invariant is difficult. The following are examples of spaces for which we know some estimations:

- Assume that $Y$ is a finite or infinite dimensional Hadamard manifold or an $\mathbb{R}$-tree. Then we have $\delta(Y)=0$.

- Assume that $Y_{p}$ is the building $P S L\left(3, \mathbb{Q}_{p}\right) / P S L\left(3, \mathbb{Z}_{p}\right)$. Then $\delta\left(Y_{p}\right) \geq$ $\frac{(\sqrt{p}-1)^{2}}{2(p-\sqrt{p}+1)}$. When $p=2$, we have $\delta\left(Y_{2}\right) \leq 0.4122 \ldots$ 
In [13, the second author obtained a geometric condition for $\delta$ to be bounded from above by a constant less than 1 . Kondo [8] constructed the first example of a complete $\operatorname{CAT}(0)$ space with $\delta=1$

Although the Izeki-Nayatani invariant is defined as a global invariant of the space, it can be estimated by the local property of the space. To see this, we define the following notation, which is introduced in 7 .

Definition 2.3. Let $Y$ be a complete $\operatorname{CAT}(0)$ space, and $O \in Y$. We define $\delta(Y, O) \in[0,1]$ to be

$$
\delta(Y, O)=\sup \{\delta(\nu) \mid \nu \in \mathcal{P}(Y), \operatorname{bar}(\nu)=O\} .
$$

If no such $\nu$ exists, we define $\delta(Y, O)=0$.

The following lemma is basic (see [14, Lemma 3.3]).

Lemma 2.4. Suppose that $Y$ is a complete $\mathrm{CAT}(0)$ space. Then we have

$$
\delta(Y)=\sup \left\{\delta\left(T C_{p} Y, O\right) \mid p \in Y\right\}=\sup \left\{\delta\left(T C_{p} Y\right) \mid p \in Y\right\} .
$$

We recall the following definition from [6].

Definition 2.5. Let $T$ be a metric cone with the origin $O_{T}$. Let $D_{\mathrm{rad}}(T)$ be the infimum of $D$ satisfying the following condition: there exists a map $f: T \rightarrow \mathcal{H}$ to a Hilbert space $\mathcal{H}$ such that

$$
\|f(v)\|=d_{T}\left(O_{T}, v\right)
$$

and

$$
\frac{1}{D} d_{T}(v, w) \leq\|f(v)-f(w)\| \leq d_{T}(v, w)
$$

for all $v, w \in T$. This number $D_{\mathrm{rad}}(T)$ is called the radial distortion of $T$. If there exists no such $f$, we define $D_{\text {rad }}(T)=\infty$.

Izeki, Kondo and Nayatani [6] proved the following relation between this invariant and $\delta$.

Lemma 2.6 ([6]). Let $T$ be a complete $\operatorname{CAT}(0)$ metric cone with the origin $O_{T}$. Then we have

$$
\delta\left(T, O_{T}\right) \leq 1-\frac{1}{D_{\text {rad }}(T)^{2}} .
$$

We will estimate the radial distortion of each tangent cone of $\operatorname{CAT}(0)$ cube complexes in the next section.

\section{Embeddings of tangent CONes of Cube COMPleXes}

In this section, we prove Theorem 1.4. Let $C$ be the (metric) product of half lines $\ell_{1}, \ldots, \ell_{n}$ such that each $\ell_{i}=[0, \infty)$. For each nonempty subcollection of $\{1, \ldots, n\},\left\{i_{1}<i_{2}<\cdots<i_{m}\right\}$, we define a subset, called a face of dimension $m$ of $C$, by

$$
\{0\} \times \cdots \times\{0\} \times \ell_{i_{1}} \times\{0\} \times \cdots \times\{0\} \times \ell_{i_{m}} \times\{0\} \times \cdots \times\{0\},
$$

where all coordinates other than $i_{j}$ are 0 . (This is like a face of a cube.) We say that the point $0 \times \cdots \times 0$ is the face of dimension 0 . 
Lemma 3.1 (cube lemma). Let $C$ be a finite product of half-lines. Let $X$ be the union of several faces of $C$. Denote the path metric in $X$ by $d_{X}$, and let $d_{C}$ be the (path) metric in $C$. Then for any points $p, q \in X$,

$$
d_{X}(p, q) / \sqrt{2} \leq d_{C}(p, q) \leq d_{X}(p, q) .
$$

Proof. $d_{C}(p, q) \leq d_{X}(p, q)$ is clear. So we show the other inequality. There is a unique face $P$ of $C$ which has the least dimension among faces which contain $p$. Also, there is a unique face $Q$ for $q$. Let $R=P \cap Q$ be the intersection, which is also a face. Let $p^{\prime}, q^{\prime} \in R$ be the projections of $p, q$, respectively. Look at the path

$$
\left[p, p^{\prime}\right] \cup\left[p^{\prime}, q^{\prime}\right] \cup\left[q^{\prime}, q\right] \subset(P \cup Q) \subset X .
$$

The angle at $p^{\prime}, q^{\prime}$, measured in $C$, is $\pi / 2$, and therefore we compute

$$
d_{C}(p, q)^{2}=d_{C}\left(p, p^{\prime}\right)^{2}+d_{C}\left(p^{\prime}, q^{\prime}\right)^{2}+d_{C}\left(q^{\prime}, q\right)^{2} .
$$

On the other hand, to estimate $d_{X}(p, q)$, we compute $d_{P \cup Q}(p, q)$. Let us look for the shortest path among the paths from $p$ to $q$ in $P \cup Q$ which starts from $p$ then reaches some point $r \in\left[p^{\prime}, q^{\prime}\right]$, then goes to $q$. (Imagine for example that $P$ is a 3 -cube and $Q$ is a square such that $P$ and $Q$ share an edge, which is $R$.) We then find a path, the square of whose length is

$$
d_{C}\left(p^{\prime}, q^{\prime}\right)^{2}+\left(d_{C}\left(p, p^{\prime}\right)+d_{C}\left(q, q^{\prime}\right)\right)^{2} .
$$

This number gives an upper bound of $d_{X}(p, q)^{2}$. On the other hand, this number is $\leq 2 d_{C}(p, q)^{2}$ by the above computation, so that $d_{X}(p, q)^{2} \leq 2 d_{C}(p, q)^{2}$.

Lemma 3.2. Let $X$ be a $\mathrm{CAT}(0)$ cube complex and $p$ a vertex. Then there exists a 1-Lipschitz map $\phi: T C_{p}(X) \rightarrow \mathcal{H}$ such that

$$
d_{\mathcal{H}}(0, \phi(v))=d_{T C_{p} X}(O, v), \quad d_{T C_{p} X}(u, v) / \sqrt{2} \leq d_{\mathcal{H}}(\phi(u), \phi(v)) \leq d_{T C_{p} X}(u, v)
$$

for all $u, v \in T C_{p} X$.

Proof. Let $e_{i}(i \in I)$ be the set of all edges of $X$ which contain $p$. For each $n$-cube $C$ in $X$ which contains $p$, any point $x \in C$ is uniquely written as $x=\sum_{i}^{n} t_{i} e_{j_{i}}$ such that $e_{j_{i}}$ are the edges which are contained in $C$, and $1 \geq t_{i} \geq 0$. In this way, any point $x \in C$ is uniquely written as $x=\sum_{i} t_{i} e_{i}$ such that $1 \geq t_{i} \geq 0$ and $t_{i}=0$ except for finitely many ones. Similarly, any point $x \in T C_{p} X$ is uniquely written as

$$
x=\sum_{i} t_{i} e_{i}
$$

where $0 \leq t_{i} \leq 1$ and $t_{i}=0$ except for finitely many ones. To be precise we have abused the notation such that $e_{i}$ is now the unit vector in $T C_{p} X$ which corresponds to the direction of the edge $e_{i}$. Now prepare a set of orthonormal vectors $v_{i} \in \mathcal{H}(i \in I)$. To define a map $\phi: T C_{p} X \rightarrow \mathcal{H}$, for $x=\sum_{i} t_{i} e_{i}$, set $\phi(x)=\sum_{i} t_{i} v_{i}$. Now apply Lemma 3.1 .

Remark 3.3. In the proof of the above lemma, we use the following condition which is satisfied at each vertex $v$ of a $\operatorname{CAT}(0)$ cube complex $X$ : Let $e_{i}(i \in I)$ be the collection of all edges which contain $v$. For each finite subset $J \subset I$, there is at most one cube in $X$ which contains all of the $e_{j}, j \in J$ and whose dimension is $|J|$. 
Thus, we have proved the following proposition.

Proposition 3.4. Let $Y$ be a $\mathrm{CAT}(0)$ cube complex, and $p \in Y$. Then we have

$$
D\left(T C_{p} Y\right) \leq \sqrt{2}, \quad D_{\text {rad }}\left(T C_{p} Y\right) \leq \sqrt{2} .
$$

Proof. Notice that we may assume without loss of generality that $p$ is a vertex. This is because if $p$ is not a vertex, namely, $p$ is an interior point of an $n$-dimensional cube, then there exists another $\operatorname{CAT}(0)$ cube complex $X^{\prime}$ and a vertex $p^{\prime}$ such that a neighborhood of $p$ in $X$ is isometric to a neighborhood of $\left(p^{\prime}, 0\right)$ in $X^{\prime} \times \mathbb{R}^{n}$. Then, $T C_{p} X$ is isometric to $T C_{p^{\prime}} X^{\prime} \times \mathbb{R}^{n}$.

Now the proposition follows from Lemma 3.2 .

Combining Proposition 3.4 with Lemma 2.6 and Lemma 2.4, we obtain Theorem 1.4 Now Theorem 2.2 applies to CAT(0) cube complexes; therefore we obtain Theorem 1.5.

\section{WANG'S INVARIANT AND UNIFORM EMBEDDABILITY OF EXPANDERS}

In this section, we prove Corollary 1.9, For a finite graph $G$ and a complete CAT(0) space $Y$, Wang 15 defined the first positive eigenvalue of a combinatorial Laplacian, $\lambda_{1}(G, Y)$, for maps from the set of vertices of $G$ to $Y$. This is a natural generalization of the one for the usual combinatorial Laplacian, $\lambda_{1}(G)$, for realvalued functions on the set of vertices.

Definition $4.1\left(\lambda_{1}(\Gamma, Y)\right.$ by Wang $)$. Let $G=(V, E)$ be a finite graph, and $Y$ be a complete $\operatorname{CAT}(0)$ space. We assume that $Y$ contains at least two points. The Wang's invariant $\lambda_{1}(G, Y)$ is defined by

$$
\lambda_{1}(G, Y)=\inf _{\phi} \frac{\sum_{\{u, v\} \in E} d_{Y}(\phi(u), \phi(v))^{2}}{\sum_{v \in V} \operatorname{deg}(v) d_{Y}(\phi(v), \bar{\phi})^{2}},
$$

where the infimum is taken over all nonconstant maps $\phi: V \rightarrow Y$, and $\bar{\phi}$ denotes the barycenter of the probability measure $\sum_{v \in V} \frac{\operatorname{deg}(v)}{2|E|} \operatorname{Dirac}_{\phi(v)}$ on $Y$. $\operatorname{Dirac}_{\phi(v)}$ is the Dirac measure at $\phi(v) \in Y$.

If we see $\mathbb{R}$ as a $\operatorname{CAT}(0)$ space, $\lambda_{1}(G)=\lambda_{1}(G, \mathbb{R})$ holds. If we take a Hilbert space $\mathcal{H}$, it is not hard to show from the definition that $\lambda_{1}(G, \mathcal{H})=\lambda_{1}(G)$. Originally, the invariant $\delta(Y)$ was introduced to give an estimate of $\lambda_{1}(G, Y)$. Izeki and Nayatani have shown the following.

Theorem 4.2 (Izeki-Nayatani). 7, Proposition 6.3] Let $G$ be a finite graph and $Y$ a complete $\mathrm{CAT}(0)$ space. Then we have

$$
(1-\delta(Y)) \lambda_{1}(G) \leq \lambda_{1}(G, Y) \leq \lambda_{1}(G) .
$$

By Theorems 1.4 and 4.2 we immediately obtain the following.

Corollary 4.3. Let $X$ be a complete CAT(0) cube complex, and $G$ be a finite graph. Then

$$
\lambda_{1}(G) / 2 \leq \lambda_{1}(G, X) \leq \lambda_{1}(G) .
$$

The invariant $\lambda_{1}(G, Y)$ is important not only in the study of fixed point theorems, but also in connection with the embedding of graphs in $Y$. To explain the result 
by Gromov that a sequence of expanders does not uniformly embed into a Hilbert space, let $\left\{G_{n}=\left(V_{n}, E_{n}\right)\right\}$ be a sequence of finite graphs such that

(1) The number of vertices of $G_{n}$ goes to infinity as $n$ goes to infinity.

(2) There exists $d>0$ such that $\operatorname{deg}(v) \leq d$ for all $v \in V_{n}$ and all $n$.

If the sequence $G_{n}$ uniformly embeds into a Hilbert space $\mathcal{H}$, then one can show that

$$
\liminf _{n} \lambda_{1}\left(G_{n}\right)=0
$$

If $\left\{G_{n}\right\}$ is a sequence of expanders, then by definition, it must satisfy

$$
\liminf _{n} \lambda_{1}\left(G_{n}\right)=c>0
$$

therefore we obtain the following.

Theorem 4.4 (Gromov, see [11). Let $\left\{G_{n}\right\}$ be a sequence of expanders. Then there is no uniform embedding of $\left\{G_{n}\right\}$ into a Hilbert space.

By Corollary 4.3, for a complete $\mathrm{CAT}(0)$ cube complex $Y$, the sequence of expanders $\left\{G_{n}\right\}$ satisfies

$$
\liminf _{n} \lambda_{1}\left(G_{n}, Y\right) \geq \liminf _{n} \lambda_{1}\left(G_{n}\right) / 2=c / 2>0 .
$$

As for the equation (4.1), the same conclusion follows from the same argument if we replace the Hilbert space by a complete $\mathrm{CAT}(0)$ space $Y$. A similar observation can be found in [8] and [9] as we mentioned. For the reader's convenience, we record the argument.

Theorem 4.5. Let $\left\{G_{n}=\left(V_{n}, E_{n}\right)\right\}$ be a sequence of finite graphs satisfying the conditions (1) and (2). If $\left\{G_{n}\right\}$ embeds uniformly into a complete CAT(0) space $Y$, then we have

$$
\liminf _{n \rightarrow \infty} \lambda_{1}\left(G_{n}, Y\right)=0 .
$$

Proof. Let $\left\{f_{n}: G_{n} \rightarrow Y\right\}$ be a uniform embedding and $\rho_{1}, \rho_{2}:[0, \infty) \rightarrow[0, \infty)$ be unbounded nondecreasing functions such that

$$
\rho_{1}\left(d_{G_{n}}\left(x, x^{\prime}\right)\right) \leq d_{Y}\left(f_{n}(x), f_{n}\left(x^{\prime}\right)\right) \leq \rho_{2}\left(d_{G_{n}}\left(x, x^{\prime}\right)\right),
$$

for all $x, x^{\prime} \in G_{n}$ and all $n$. Put $c=\rho_{2}(1)$. Then we have $d\left(f_{n}(v), f_{n}(w)\right) \leq c$ for all $\{v, w\} \in E_{n}$ and all $n$.

First, observe that for any $r>0$, the preimage of an $r$-ball has diameter at most $\rho_{1}^{-1}(2 r)$. Thus the preimage of any $r$-ball in $Y$ by any $f_{n}$ contains at most $d^{1+\rho_{1}^{-1}(2 r)}$ vertices of $G_{n}$ since $G_{n}$ satisfies the property (2).

Next, by the definition of $\lambda_{1}\left(G_{n}, Y\right)$, if $\lambda_{1}\left(G_{n}, Y\right)>0$ we have

$$
\sum_{v \in V_{n}} d_{Y}\left(f_{n}(v), \overline{f_{n}}\right)^{2} \leq \frac{1}{\lambda_{1}\left(G_{n}, Y\right)} \sum_{\{v, w\} \in E_{n}} d_{Y}\left(f_{n}(v), f_{n}(w)\right)^{2} .
$$

The right-hand side is no greater than $\frac{1}{\lambda_{1}\left(G_{n}, Y\right)} \frac{d\left|V_{n}\right|}{2} c^{2}$. Thus at least $\frac{\left|V_{n}\right|}{2}$ terms in the sum on the left-hand side are at most $\frac{1}{\lambda_{1}\left(G_{n}, Y\right)} c^{2} d$. This means that the preimage of the ball of radius $\left(\frac{d}{\lambda_{1}\left(G_{n}, Y\right)}\right)^{\frac{1}{2}} c$ centered at $\overline{f_{n}} \in Y$ contains at least $\frac{\left|V_{n}\right|}{2}$ vertices of $G_{n}$. 
To argue by contradiction we assume that

$$
\liminf _{n} \lambda_{1}\left(G_{n}, Y\right)=\lambda>0 .
$$

Then there exists an arbitrarily large $n$ such that $\lambda_{1}\left(G_{n}, Y\right)>\frac{\lambda}{2}$. Put $r=\left(\frac{2 d}{\lambda}\right)^{\frac{1}{2}} c$. The above discussion implies that for such an $n$ the preimage of the $r$-ball centered at $\overline{f_{n}}$ by $f_{n}$ contains at least $\frac{\left|V_{n}\right|}{2}$ vertices of $G_{n}$. But then $\frac{\left|V_{n}\right|}{2} \leq d^{1+\rho_{1}^{-1}(2 r)}$, which is impossible.

Now, Corollary 1.9 follows immediately from (4.2) and Theorem 4.5

\section{REFERENCES}

[1] M. R. Bridson and A. Haefliger. Metric spaces of non-positive curvature, volume 319 of Grundlehren der Mathematischen Wissenschaften. Springer-Verlag, Berlin, 1999. MR:1744486 (2000k:53038)

[2] J. Brodzki, S. J. Campbell, E. Guentner, G. A. Niblo, and N. J. Wright. Property A and CAT(0) cube complexes. J. Funct. Anal., 256(5):1408-1431, 2009. MR22490224(2010i:20044)

[3] P. de la Harpe and A. Valette. La propriété $(T)$ de Kazhdan pour les groupes localement compacts. Astérisque 175:158, 1989. MR1023471 (90m:22001)

[4] M. Gromov. Random walk in random groups. Geom. Funct. Anal., 13(1):73-146, 2003. MR.1978492 (2004j:20088a)

[5] H. Izeki, T. Kondo, and S. Nayatani. Fixed-point property of random groups. Ann. Global Anal. Geom., 35(4):363-379, 2009. MR2506240 (2010d:20050)

[6] H. Izeki, T. Kondo, and S. Nayatani. $N$-step energy of maps and fixed-point property of random groups. Groups Geom. Dyn., to appear.

[7] H. Izeki and S. Nayatani. Combinatorial harmonic maps and discrete-group actions on Hadamard spaces. Geom. Dedicata, 114:147-188, 2005. MR 2174098 (2006k:58024)

[8] T. Kondo. CAT(0) spaces and expanders. Math. Z., to appear.

[9] M. Mendel and A. Naor. Towards a Calculus for Non-Linear Spectral Gaps [Extended Abstract]. Proceedings of the Twenty-First Annual ACM-SIAM Symposium on Discrete Algorithms, 236-255, 2010.

[10] G. Niblo and L. Reeves. Groups acting on CAT(0) cube complexes. Geom. Topol., 1:1-7, 1997. MR1432323(98d:57005)

[11] J. Roe. Lectures on Coarse Geometry, volume 31 of University Lecture Series. American Mathematical Society, Providence, RI, 2003. MR2007488 (2004g:53050)

[12] L. Silberman. Addendum to: "Random walk in random groups". Geom. Funct. Anal., 13(1):147-177, 2003. MR1978493 (2004j:20088b)

[13] T. Toyoda. CAT(0) spaces on which a certain type of singularity is bounded. Kodai Math. J., 33:398-415, 2010. MR2754329

[14] T. Toyoda. Fixed point property for a CAT(0) space which admits a proper cocompact group action. Preprint, 2010. arXiv:1102.0729

[15] M.-T. Wang. Generalized harmonic maps and representations of discrete groups. Comm. Anal. Geom., 8(3):545-563, 2000. MR1775138(2001m:58039)

Graduate School of Information Science, Tohoku University, Aoba-ku, Sendai, MIYAGI, 980-8579, JAPAN

E-mail address: fujiwara@math.is.tohoku.ac.jp

Graduate School of Mathematics, Nagoya University, Chikusa-Ku, Nagoya, 464-8602, JAPAN

E-mail address: tetsu.toyoda@math.nagoya-u.ac.jp 